\title{
Miniaturized Planar Implanted Spiral Antenna Inside the Heart Muscle at MICS Band for Future Leadless Pacemakers
}

\author{
Mehrab Ramzan ${ }^{1}$, Xiao Fang ${ }^{1}$, Qiong Wang ${ }^{1}$, Niels Neumann ${ }^{1}$, Dirk Plettemeier ${ }^{1}$ \\ ${ }^{1}$ Chair of RF Engineering, Communication laboratory, TU Dresden, Dresden, Germany
}

\begin{abstract}
This paper presents a miniaturized planar spiral implanted antenna inside the heart tissue for future leadless pacemakers. The implanted antenna is designed for Medical Implant Communication Service (MICS) band (402-405 MHz). The surrounding medium permittivity and tangent loss effect on the implantable antennas are also investigated. Moreover, the forward transmission coefficient between two implanted spiral antenna is analyzed. The investigation has been carried out using EM full-wave simulations.
\end{abstract}

Index Terms:- Implanted antennas, Spiral antenna, transmission efficiency, Heart Muscle, Lossy medium, pacemaker, MICS band, Phantoms, Body area communication.

\section{INTRODUCTION}

In the past years, miniaturized future leadless pacemakers have gained great attention [1-3]. These kinds of small self contained devices can replace the widely used conventional wired pacemakers which have infection issues and lead complexities. For the effective monitoring of the heart functions, there should be at least two leadless pacemakers in different chambers of the human heart muscle as shown in Fig 1 [4]. However, the RF propagation losses are inevitable due to the tissue properties of the human heart (higher water content), which lead to a difficulty in effective communication between these leadless pacemakers. The human heart muscle permittivity decreases and conductivity increases with respect to the frequency. On the other hand, there is a high demand for shrinking the size of the electronic components. The antenna is a key element of wireless communication and its size is directly related to the wavelength. However, the size reduction at lower frequencies, where human conductivity is less, turns challengeable and leads to a tremendous reduction of the antenna efficiency. In this paper, we propose a miniaturized spiral antenna for the Medical Implant Communication Service (MICS) band (402-405 MHz) where the propagation losses are much less as compared to the other potential frequencies. Initially, we meet the reduction of the spiral antenna size by using longer path structures. However, the impedance matching becomes challenging due to the lossy nature of the surrounding human heart tissue and the longer wavelengths at MICS band. In order to suffice all these requirements, we introduce a shortening pin between the antenna top surface and ground plane to make the antenna resonant at MICS band.

This paper is organized as follows. It starts with a brief introduction of the planar spiral antenna and its dimensions. The second section defines the simulation model and simulated results are compared in the homogeneous and inhomogeneous heart muscle. In the third section, the influence of surrounding medium permittivity and tangent loss are investigated. Finally, a conclusion and an outlook are given.
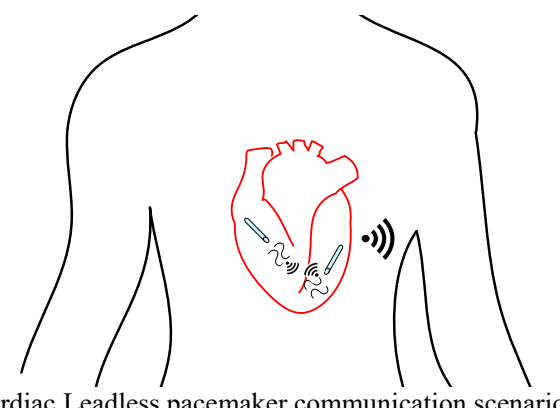

Fig 1. Intracardiac Leadless pacemaker communication scenario

\section{SPIRAL DESIGN}

The miniaturization of antennas at lower frequencies becomes challenging owing to the large size of the wavelength and surrounding lossy human tissue. The implanted antenna design is based on the spiral structure for making the antenna current paths to be longer assisting in the miniaturization of the antenna. The antenna consists of a circular planar spiral antenna at the top layer and a full circular ground plane at the bottom layer with a $50 \mathrm{ohm}$ coaxial feed point at its center. However, this technique is not enough in the lossy medium as compared to the free space case rendering the task more difficult. In order to further assist the miniaturization of the planar spiral antenna, a shortening pin is introduced between the top and bottom layer as shown in Fig 2. Fig 2 (c) shows the side view of the planar spiral antenna manufactured on the top layer of Rogers RO3210 substrate with $\varepsilon_{\mathrm{r}}$ of 10.2 and a loss tangent of 0.003 . In order to further shrink the size of the conventional circular spiral antenna and for matching in the lossy medium a superstrate of Rogers 3006 with $\varepsilon_{\mathrm{r}}$ of 6.4 is used on top. This superstrate does not only help in impedance matching, but it also plays a vital role as an insulating layer which is critical for the implanted antenna in order to preserve the current distribution on the surface of the antenna for proper radiation. Table 1 shows the dimensions of the miniaturized implanted spiral antenna where the radius of the antenna is 5 $\mathrm{mm}$ in MICS frequency band.

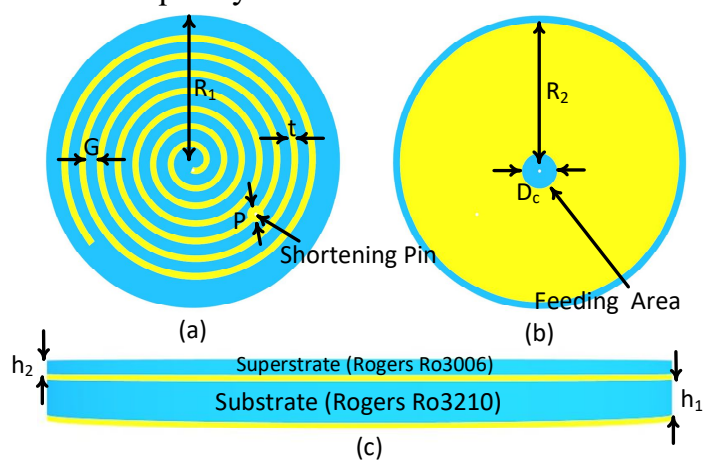

Fig 2. Miniaturized Implanted Spiral antenna (a) Top view (b) Bottom View (c) Side view of the antenna 
TABLE I. DIMENSIONS OF MINIATURIZED SPIRAL ANTENNA

\begin{tabular}{c|c} 
Parameters & Dimensions (mm) \\
\hline \hline $\mathrm{R}_{1}$ & 5 \\
\hline $\mathrm{R}_{2}$ & 4.75 \\
\hline $\mathrm{D}_{\mathrm{c}}$ & 1.2 \\
\hline $\mathrm{T}$ & 0.2 \\
\hline $\mathrm{G}$ & 0.3 \\
\hline $\mathrm{P}$ & 0.6 \\
\hline $\mathrm{h}_{1}$ & 0.64 \\
\hline $\mathrm{h}_{2}$ & 0.25
\end{tabular}

\section{SimUlATED RESUlTS IN HOMOGENEOUS AND INHOMOGENEOUS HEART}

The human heart in reality is an inhomogeneous medium surrounded by different tissues possessing different electrical properties. Therefore, it is necessary to assign proper boundary conditions to the lossy medium during the EM full-wave simulation in order to reduce the simulation time. In the first step, the implanted spiral antenna is simulated inside a homogeneous sphere having a radius of $4 \mathrm{~cm}$ as shown in Fig 3 . Then, the implanted antenna is simulated inside an inhomogeneous medium surrounded by fat, skin, and air for reference and comparison as shown in Fig 4. In both of the cases, the relative permittivities and conductivities of tissues are assigned according to the 4-cole-cole model expression proposed by Gabriel [5].

$$
\dot{\varepsilon}_{r}(\omega)=\varepsilon_{\infty}+\sum_{n=1}^{4} \frac{\Delta \varepsilon_{n}}{1+\left(j \omega \tau_{n}\right)^{1-\alpha_{n}}}+\frac{\sigma_{0}}{j \omega \varepsilon_{0}}
$$

Each of the above terms is described as a modified Debye expression because $\alpha_{n}$ is introduced to describe the deviation from Debye behavior. All the corresponding parameters in Equ. (1) for obtaining the complex permittivities of heart, fat, and skin are provided by [6]. Table II gives the electrical properties and dimensions of used tissues. Fig $\mathbf{5}$ shows the simulated results of the implanted spiral antenna inside the homogeneous and inhomogeneous medium. Both of the curves show the same behavior because the antenna is enclosed mostly by the heart tissue. Fig 6 shows the impedance of the proposed spiral antenna inside the lossy human heart tissue. The spiral antenna shows a parallel and a series resonance which is produced with the help of a shortening pin and the real part of the impedance is reduced with the help of using a superstrate.

TABLE II. TISSUE PROPERTIES AND DIMENSIONS USED FOR THE SIMULATION

\begin{tabular}{|c|c|c|c|}
\hline Parameters & $\begin{array}{c}\text { Relative } \\
\text { Permittivity }\end{array}$ & $\begin{array}{c}\text { Conductivity } \\
(\mathbf{S} / \mathbf{m})\end{array}$ & $\begin{array}{c}\text { Dimension } \\
(\mathbf{m m})\end{array}$ \\
\hline Heart Muscle & 66 & 0.966 & $\mathrm{R}_{\mathrm{h}}=40$ \\
\hline Fat & 5.578 & 0.0411 & $\mathrm{R}_{\mathrm{F}}=50$ \\
\hline Skin & 46.71 & 0.689 & $\mathrm{R}_{\mathrm{S}}=60$ \\
\hline
\end{tabular}

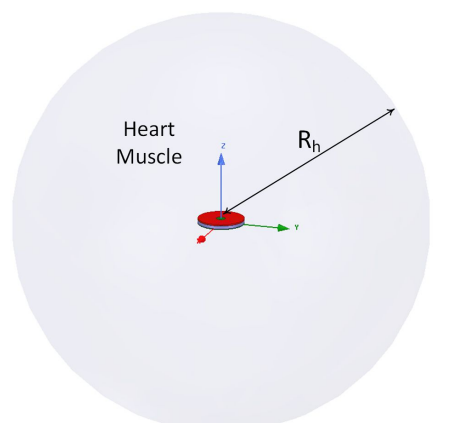

Fig 3. Simulation model of the implanted spiral antenna in the homogeneous heart muscle.

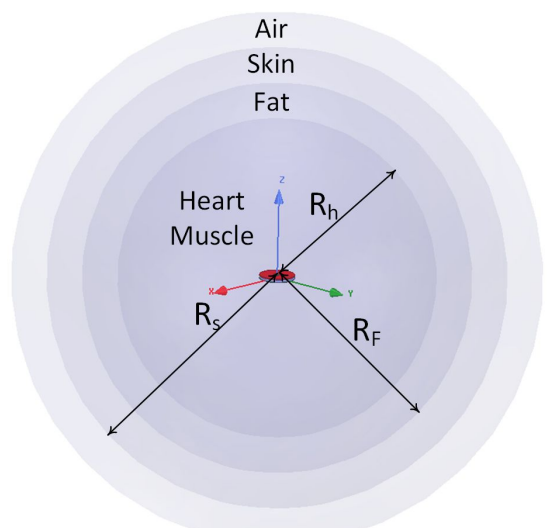

Fig 4. Simulation model of the implanted spiral antenna in inhomogeneous heart muscle.

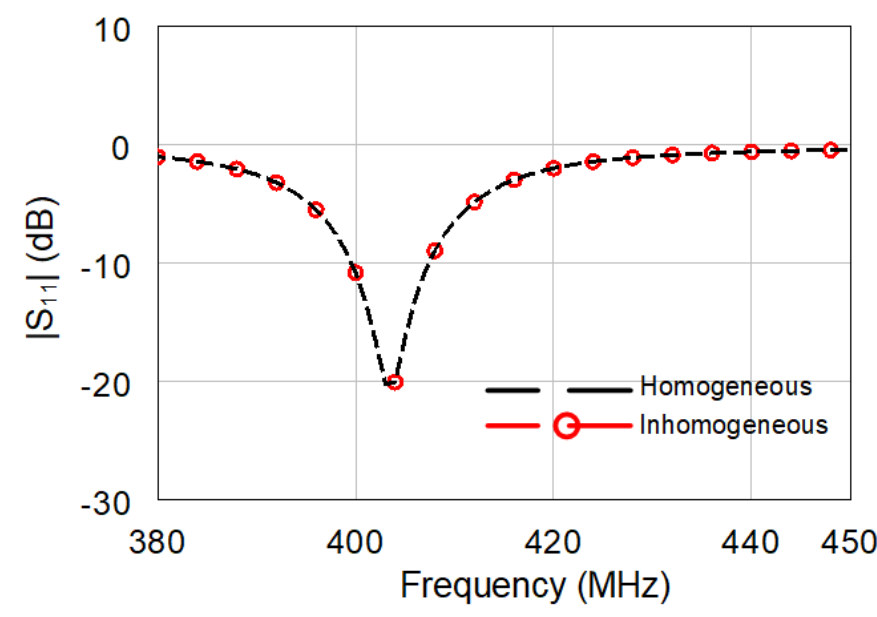

Fig 5. Simulated results of the miniaturized planar spiral antenna in homogeneous and inhomogeneous medium of heart muscle.

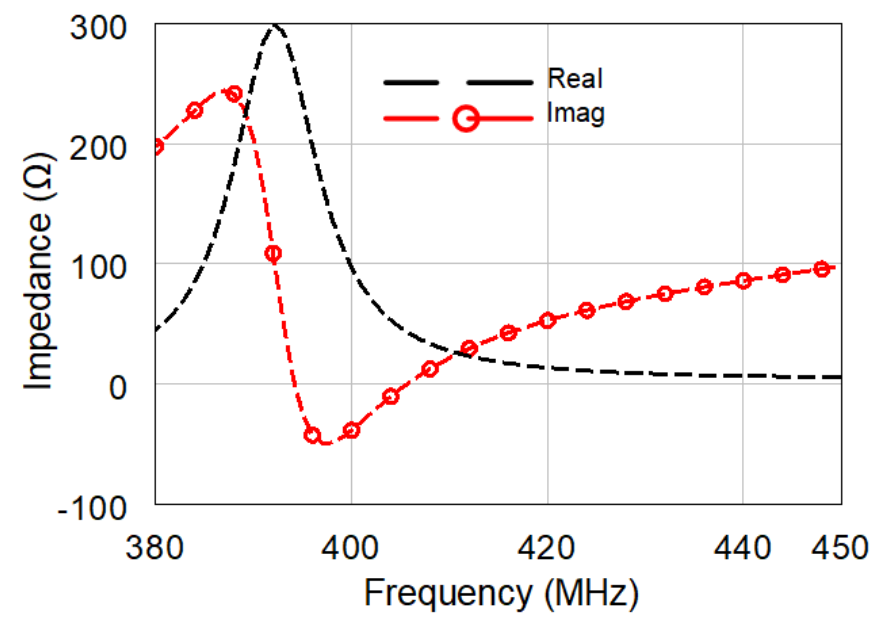

Fig 6. Simulated real and imaginary part of the miniaturized spiral antenna.

\section{INFLUENCE OF SURROUNDING MEDIUM ON THE IMPLANTABLE ANTENNA}

In this section, an in-depth investigation is carried out to check the influence of the surrounding medium on the implantable antenna characteristic. Initially, the influence of the permittivity of the surrounding medium is analyzed. The medium losses are kept constant with the value of heart tissue and the relative permittivity is varied from 40 to 70 . Fig 7 shows that there is a slight change in the bandwidth when the relative permittivity is around 40 . In terms of the real part of the 
impedance, the varying permittivity of the medium reduces the maximum real impedance of the spiral antenna as shown in Fig 8. The imaginary part of the impedance illustrated in Fig. 9, also shows the same decreasing behavior and the slope of the resonance is decreasing at lower values of permittivity. As a result, the implanted antenna is showing wider bandwidth at lower values of permittivity of the medium.

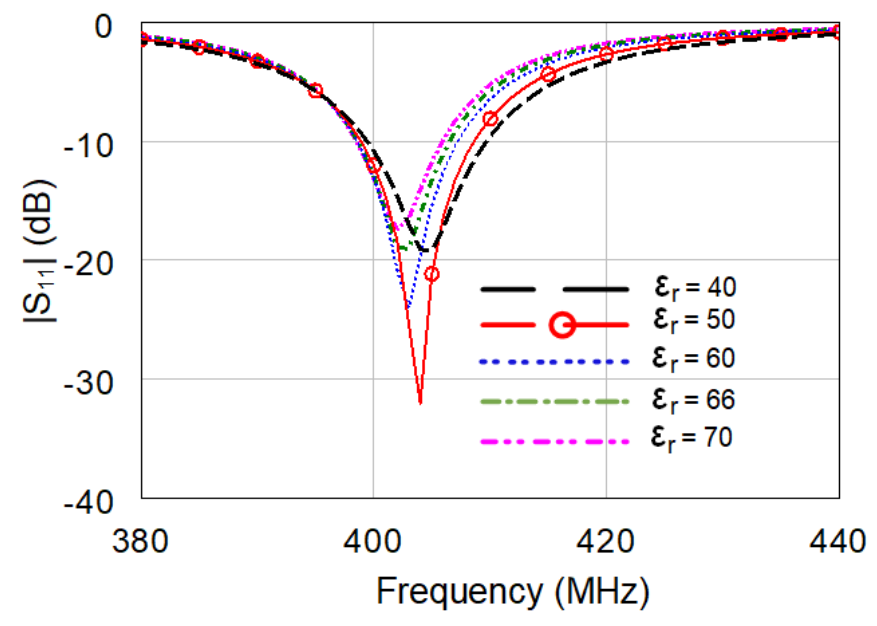

Fig 7. Simulated influence of the permittivity of the surrounding lossy medium

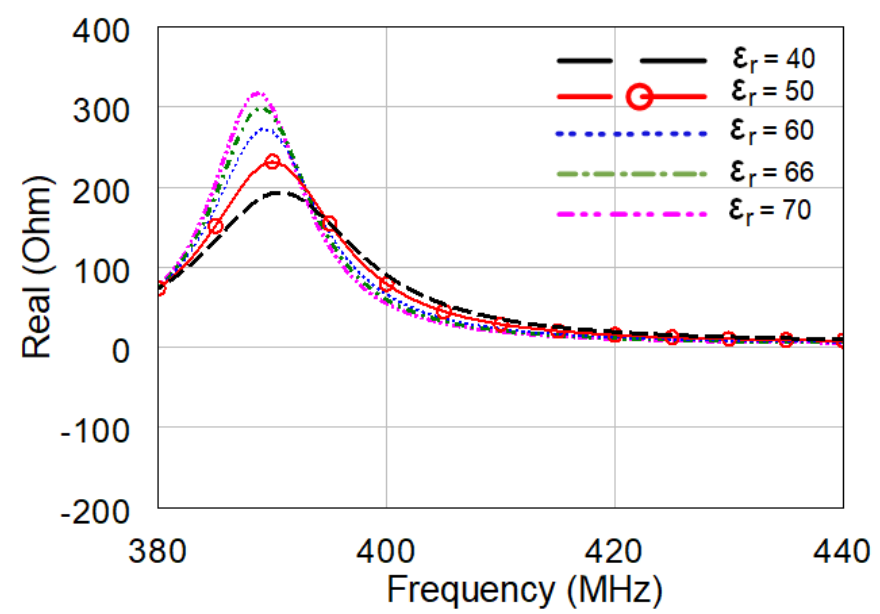

Fig 8 . Simulated real part of impedance with different permittivity values of the surrounding lossy medium

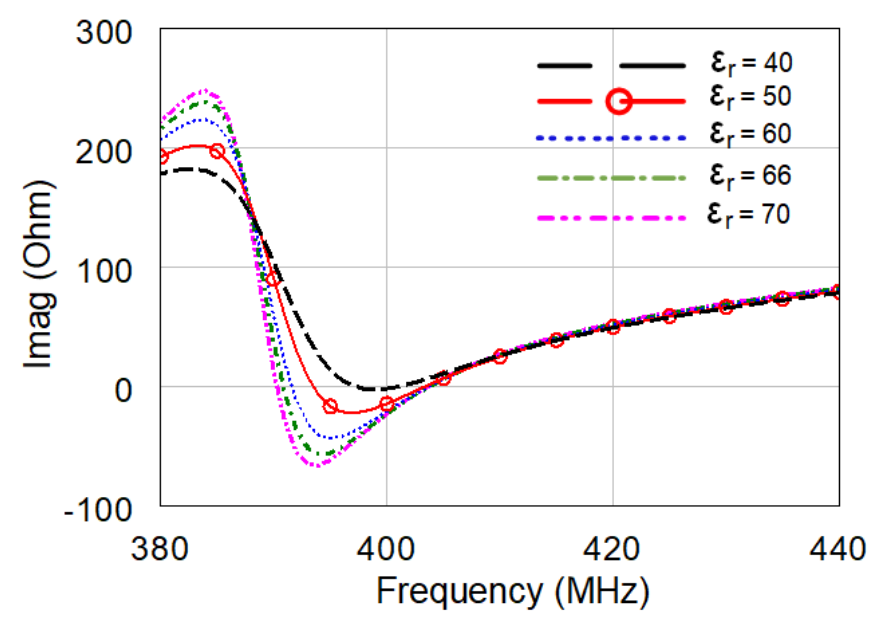

Fig 9. Simulated imaginary part of impedance with different permittivity values of the surrounding lossy medium

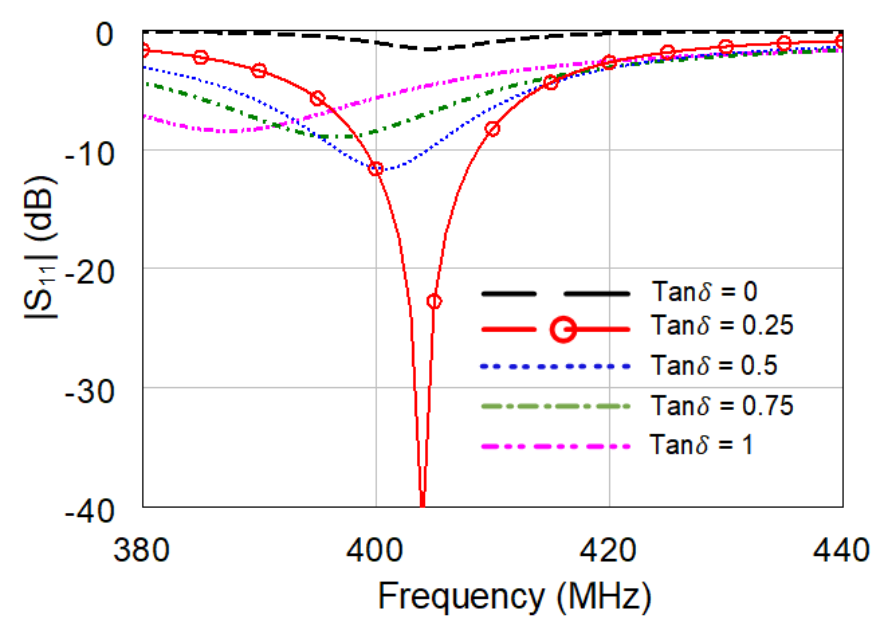

Fig 10. Simulated influence of the loss tangent of the medium on the implanted antenna

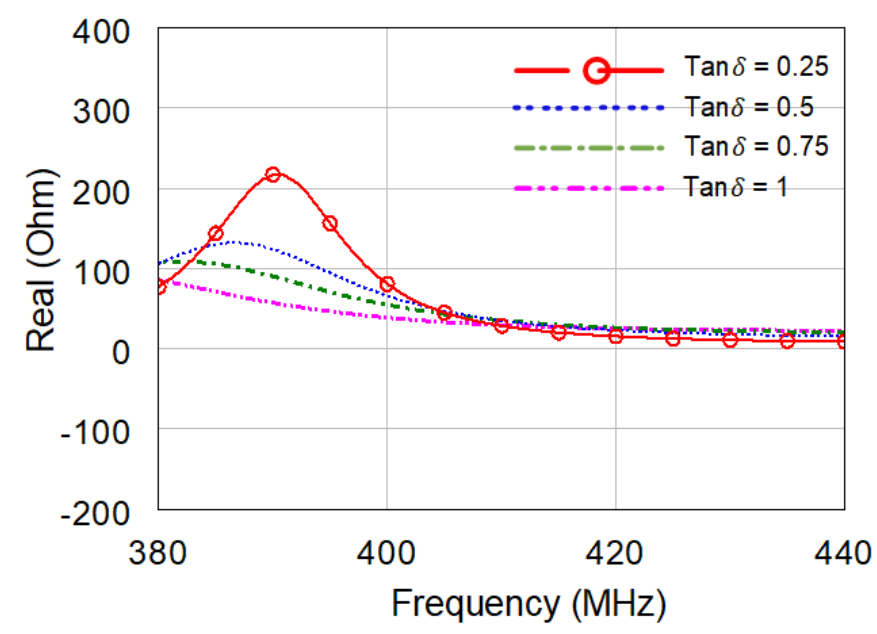

Fig 11. Simulated real part of impedance of the implanted spiral antenna at different loss tangent of the medium

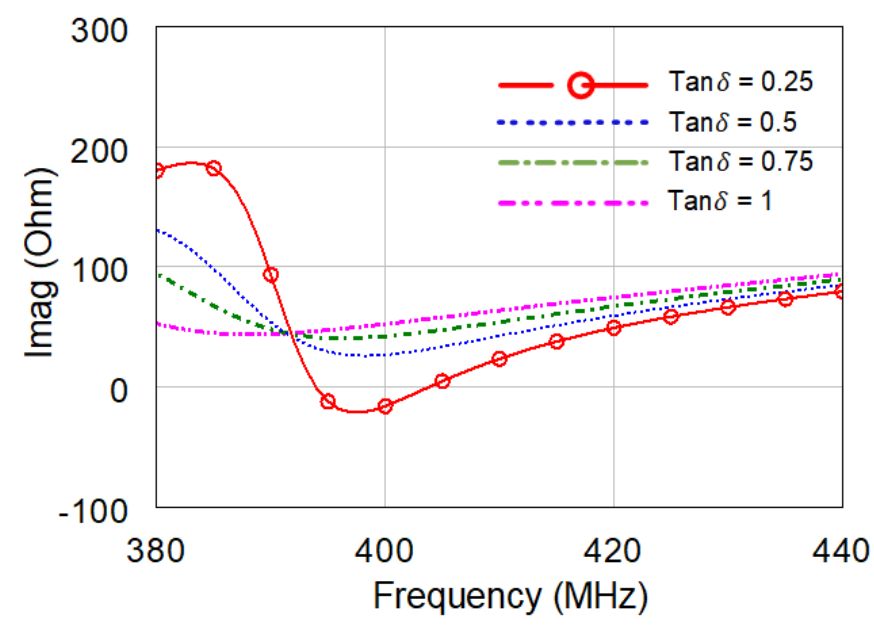

Fig 12. Simulated imaginary part of impedance of the implanted spiral antenna at different loss tangent of the medium

Further analysis is performed on the impact of the surrounding medium losses where the loss tangent value is swept from 0 to 1 . Fig. 10 shows that the loss tangent values change the magnitude of the reflection coefficient. In terms of the real part of the impedance, the maximum value decreases with increasing losses, but the value of real impedance around $403 \mathrm{MHz}$ remains the same as shown in Fig. 11. While the 
magnitude of the imaginary part of impedance shows that the resonance behavior of the spiral antenna is disappearing as the tangent loss is increasing as depicted in Fig. 12.

Fig. 13 illustrates the current distribution of the miniaturized spiral antenna with shortening pin at $403 \mathrm{MHz}$, which implies how well the power being radiated inside the lossy medium. The current is minimum at the center and starts to increase at the end turns of the spiral antenna. The transmission coefficient is also simulated at different separation distance between two implanted antennas inside the homogeneous heart tissue. The simulation model is presented in Fig. 14. The computation of transmission coefficient values clearly tells how efficiently the implanted antenna is designed to work inside the lossy heart tissue. For this purpose, the separation distance between the implanted antenna is changed from $30 \mathrm{~mm}$ to $100 \mathrm{~mm}$ and the corresponding values of the transmission coefficient vary from $-60 \mathrm{~dB}$ to $-97 \mathrm{~dB}$ as shown in Fig. 15. This implies that these antennas are having acceptable values of transmission and can be used for the data transfer during the health monitoring of the heart. In the lossy medium comparison of the implanted antenna is quite tricky and needs proper care. It requires the same surrounding and the position of the antenna inside the medium. These results can be used in the future as a reference for designing more efficient implanted antennas and comparing them in the same EM simulation environment.

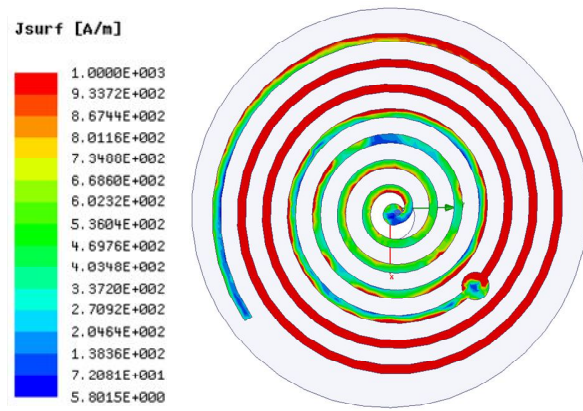

Fig 13. Surface current distribution of miniaturized spiral antenna with shortening pin at $403 \mathrm{MHz}$.

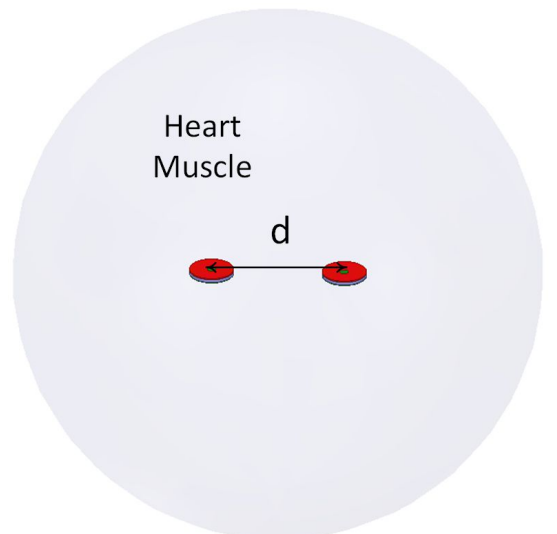

Fig 14. Simulation model for extracting the coupling coefficient of the implanted spiral antenna in the homogeneous heart muscle.

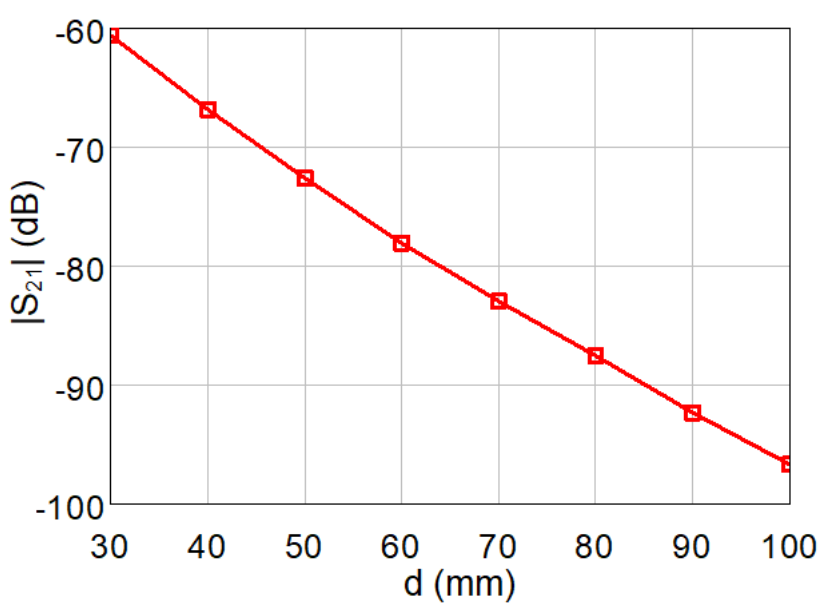

Fig 15. Transmission coefficient at different separation distance between two implanted antennas.

\section{CONCLUSION}

In conclusion, a miniaturized planar spiral implantable antenna is designed and investigated. A detailed study of surrounding medium influence on the antenna is carried out in terms of reflection coefficient, real and imaginary part of the impedance. These results help to implantable antennas and give insight on how to increase the bandwidth of the miniaturized implantable antennas. The coupling between two same kinds of implantable antennas inside the heart tissue is simulated which gives information about how efficiently the implanted antenna is designed inside the lossy medium. As a future work, these antennas will be fabricated and examined in in-vitro and in-vivo experiments.

\section{ACKNOWLEDGMENT}

This work is supported by "WIBEC" (Wireless In-Body environment) project funded by the European Union framework of Marie Curie Horizon 2020 research and innovation program under the grant 675353 .

\section{REFERENCES}

[1] D. G. D. Rocca, C. Gianni, L. D. Biase, A. Natale, A. Al-Ahmad, "Leadless Pacemakers: State of the Art and Future Perspectives," Cardiac Electrophysiology Clinics, vol. 10, no. 1, pp. 17-29, 2018,

[2] Merkel M, Grotherr P, Radzewitz A, Schmitt C. Leadless Pacing: Current State and Future Direction. Cardiol Ther. 2017;6(2):175-181.

[3] Theofanie Mela, Jagmeet P. Singh; Leadless pacemakers: leading us into the future?, European Heart Journal, Volume 36, Issue 37, 1, pp 2520-2522, October 2015.

[4] S. Sideris, S. Archontakis, P. Dilaveris, K. A. Gatzoulis, K. Trachanas, I. Sotiropoulos, P. Arsenos, D. Tousoulis, and I. Kallikazaros, "Leadless Cardiac Pacemakers: Current status of a modern approach in pacing," Hellenic Journal of Cardiology, vol. 58, no. 6, pp. 403-410, May 2017

[5] Gabriel, Sami, R. W. Lau, and Camelia Gabriel. "The dielectric properties of biological tissues: II. Measurements in the frequency range $10 \mathrm{~Hz}$ to $20 \mathrm{GHz}$." Physics in medicine \& biology, vol. 41, no. 11, pp. 2251, 1996.

[6] Gabriel, Sami, R. W. Lau, and Camelia Gabriel. "The dielectric properties of biological tissues: III. Parametric models for the dielectric spectrum of tissues." Physics in Medicine \& Biology, vol, 41, no. 11, pp. 2271, 1996. 